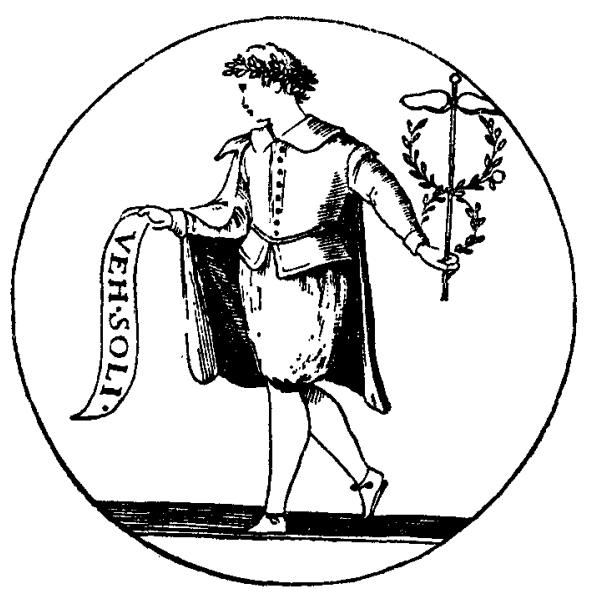

TYPOGRAPHIE PLON

$\&$ rue Garanciere

P A R I S

Le gérant : Claudr DA Cunha 


\title{
THE AMERICAN SOCIOLOGICALL ASSOCIATION
}

\author{
announces \\ the acquisition of \\ The Joumal of \\ Educational Sociology \\ from the Payne Educational \\ Sociology Foundation \\ To be published in October 1963 \\ under Association auspices as a \\ Quarterly Journal \\ SOCIOLOGY \\ $\mathrm{OF}$

\section{EDUCATION}

Sociology of Education will be devoted to studies of education as a social institution.

International and interdisciplinary in scope, Sociology of Education seeks to represent the increasing work in progress throughout the world, both theoretical and empirical, on school systems of complex societies.

Sociology of Education is edited by an international board of Advisory and Associate Editors representative of the study of society.

\section{Subscription rate $\$ 7.00$}

Subscriptions are now open for the

Volume year - October 1963 through June 1964

Four issues

\section{THE AMERICAN SOCIOLOGICAL ASSOCIATION}

NEW YORK UNIVERSITY

WASHINGTON SQUARE

NEW YORK 3, NEW YORK 


\section{RIVISTA INTERNAZIONALE DI SCIENZE SOCIALI}

anno LXXI - fascicolo I-II gennaio - febbraio 1963 marzo-aprile 1963

\section{SOMMARIO :}

F. Viro, Premessa.

F. Marinone, Lo sviluppo del sistema distributivo italiano.

L. CARONE, L'organizzazione della distribuzione in Italia : servizi.

L. Sant'Ambrogio, Il controllo dei costi della distribuzione.

P. SAviNI, Le nuove forme di distribuzione : $i$ grandi magazzini, $i$ magazzini a prezzo unico, $i$ supermercati e $i$ negozi a catena.

G. GALIZZI, L'organizzazione della distribuzione in Italia : l'agricoltura.

S. VACCA', Introdurione allo studio dei rapporti industria-distribuzione nei mercati dei beni di consumo.

L. FREY, Il finanziamento delle imprese agricole.

S. SANTOLI, La lotta contro l'inflazione nella recente esperienza austriaca.

Abbonamento annuo per l'Italia L. 3000.

per l'estero L. 6000 ovvero l'equivalente in valuta estera.

Abbonamento sostenitore L. 20000.

Redazione e Amministrazione : Largo A. Gemelli, $x-M I L A N O$ (Italia) 


\title{
Annales
}

\section{Économies Sociétés Civilisations}

\author{
Revue trimestrielle fondée en 1920 par \\ LUCIEN FEBVRE \& MARC BLOCH \\ Comité de direction : \\ FERNAND BRAUDEL \\ GEORGES FRIEDMANN - CHARLES MORAZÉ \\ Secrétaire du Comité : PAUL LEUILLIOT
}

$\mathrm{Au}$ sommaire du $\mathrm{n}^{0} 3-\mathrm{MAI}-\mathrm{JUIN}{ }_{1963}$

Claude Nicolet A Rome pendant la seconde guerre punique. Techniques financières et manipulations monétaires.

François Furet Pour une définition des classes inférieures à l'époque moderne.

Henri Goblot Dans l'ancien Iran, les techniques de l'eau et la grande bistoire.

ÉTUDES, CHRONIQUE DES SCIENCES SOCIALES, DÉBATS ET COMBATS, TRAVAUX EN COURS, ENQUETTES OUVERTES, NOTES CRITIQUES, COMPTES RENDUS.

Redaction : 20, rue de la Baume, PARIS-VIII'.

Administration : LIBRAIRIE ARMAND COLIN I03, bd St-Michel, PARIS-V

Compte de chèques postaux : Paris, No ${ }^{6} 67$ Abonnements : France et Union française, $29 \mathrm{~F}$; Étranger, $35 \mathrm{~F}$

Le numéro de 208 pages : $6 \mathrm{~F}$ 
Sciences et mystifications.

MAX DE CECCATTY

Pasternak, homme du passé ?

H. PELTIER-ZAMOYSKA

Poèmes de RAOUL BECOUSSE et ANNIE SALAGER

\section{LA DÉMOCRATIE \\ TECHNIQUE OU VALEUR ?}

« La République moderne ».

JEAN CONILH

Égaliser les chances.

S. M. LIPSET

Une démocratie qui reste veuve.

J. M. DOMENACH

« Le marxisme de notre temps $»$.

J. M. DOMENACH

\section{journal a plusieurs voix}

Les élections françaises et l'avenir de la gauche. - « Les dimanches de Villed'Avray 》. - Pierre Loti. - La Russie en Europe. - Dubillard. - Vatican I et Vatican II.

\section{CHRONIQUES}

La sociologie du travail.

Les origines de la barbarie.

La pensée théologique de Teilhard de Chardin.
H. HATZFELD

PHILIPPE IVERNEL

JACQUES NATANSON

\section{DOCUMENT}

Hiérarchie et bïcat d Grenoble.

\section{LIBRAIRIE DU MOIS}

ce numéro : 3,90 F

\begin{tabular}{|c|c|c|c|c|}
\hline \multirow{2}{*}{ Abonnement } & \multicolumn{2}{|c|}{ FRANCE } & \multicolumn{2}{|c|}{ AUTRE PAYS } \\
\hline & $\begin{array}{l}6 \text { mois } \\
\text { F } 22\end{array}$ & $\begin{array}{l}1 \text { an } \\
\text { f } 42\end{array}$ & $\begin{array}{l}6 \text { mois } \\
\text { F } 24\end{array}$ & $\begin{array}{l}1 \text { an } \\
F \quad 46\end{array}$ \\
\hline outien .............. & F 30 & F 60 & F 30 & F 60 \\
\hline Luxe numéroté $. . . \ldots . . . \ldots \ldots . . . . .$. & F 45 & $F 90$ & F $\mathbf{s o}$ & F 100 \\
\hline
\end{tabular}

\footnotetext{
ESPRIT - 19, rue Jacob - Paris -60. CCP Paris $1154-51$.
} 


\section{DIOGÈNE}

Revue internationale des sciences humaines

Rédacteur en chef : Roger CaIllors

$\mathrm{N}^{\circ} 42$

avril-juin $1_{963}$

\section{L'HOMME ET LA NOTION D'HISTOIRE EN ORIENT}

Roger CaILLOIS

Kojiro Yoshikawa

Jaroslav PRUSEK

Hajime NAKamura

Vadime ElISSEEFF

Joseph R. LEVENSON

Louis BAzIN

Alfred Stern

Georges GusDoRF
Temps circulaire, temps rectiligne.

L'bomme et la conception orientale de l'bistoire.

L'bistoire et l'épopée en Cbine et dans le monde occidental.

L'bistoire en Inde, en Cbine et au Japon.

L'Empire du Milieu, empire lointain, empire sans voisins.

Origines et itinéraire du confucianisme.

L'bomme et la notion d'bistoire dans l'Asie centrale turque du VIII ${ }^{\mathrm{e}}$ siècle.

\section{CHRONIQUES}

Les fictions it les mytbes en bistoire.

Pour une recherche interdisciplinaire.

\section{REDACTION ET ADMINISTRATION :}

6, rue Franklin, Paris-16•

(TRO 82-2I)

Revue trimestrielle paraissant en trois langues : anglais, espagnol et jrangais.

L'édition française est publiée par la Librairie Gallimard, 5, rue Sébastien-Bottin, Paris- $7^{\circ}$.

Les abonnements sont souscrits auprès de cette maison (C.C.P. r69-33, Paris).

Prix de vente au numéro: $5,50 \mathrm{~F}$.

Tarif d'abonnement : France, $20 \mathrm{~F}$; Etranger, 25,50 F. 


\section{ÉCOLE PRATIQUE dES HAUTES ÉTUDES - SORBONNE SIXIĖME SECTION : SCIENCES ÉCONOMIQUES ET SOCIALES \\ DIVISION DES AIRES CULTURELLES :}

Centre d'études sur.I'U.R.S.S. et les pays slaves

\section{Cahiers}

\section{du monde russe et soviétique}

Parait quatre fois par an

Comité de rédaction :

JACQUES BELLON, ALEXANDRE BENNIGSEN,

H. CARRERE D'ENCAUSSE, HENRI CHAMBRE, RENE DAVID, CLAUDE FRIOUX, BASILE KERBLAY, PAUL LEMERLE, FRANÇOIS DE LIENCOURT, ROGER PORTAL.

\section{AU SOMMAIRE DU N $N^{\circ}$ DE 1962}

\section{Etudes ,}

J. - B. DUROSELLE

Louis Barthou et le rapprochement franco-soviétique en 1934.

C. FRIOUX

Sur deux romans d'Aleksandr Grin.

A. BESANÇON

R.R. Falk (1886-1958).

Documents :

G. HAUPT

Correspondance Lénine-Huysmans (1905-1914).

Chronique:

C. KOUPERNIK

Psychiatrie soviétique. Tendances et réalisations.

Bibllographie :

A. KLIBANOV Les mouvements hérétiques en Russie du XIII• au XVI• siècle.

ABONNEMENTS

France : $33 \mathrm{~F}$. Le $\mathrm{n}^{\circ}: 9 \mathrm{~F}$.

Éd. Mouton, 45, rue de Lille. Paris-7e. C.C.P. 50-6796 Paris.

Etranger : $30 \mathrm{Fl}$. Le $\mathrm{n}^{\circ}: 8 \mathrm{Fl}$.

Éd. Mouton \& Co. Kerklaan 74, Rijswijk (ZH), Hollande. C.C.P. 47-3950 La Haye.

RÉDACTION-ADMINISTRATION

20 , rue de la Baume Paris-VIIl•. 


\section{RSPRIT est une \\ bibliothèque permanente}

Avril 52

Août-Sept. 52

Novembre 52

Décembre 52

Mai 53

Juin 53

Août 53

Oct.-Nov. 53

Janvier 54

Juin 54

Mars 55

Avril 55

Sept.-Oct. 55

Octobre 56

Décembre 56

Février 57

Mai 57

Juin 57

Décembre 57

Mars 58

Mai 58

Juill.-Août 58

Septembre 58

Octobre 58

Décembre 58

Janvier 59

Mars 59

Mai 59

Septembre 59

Octobre 59

Décembre 59

Janvier 60

Mars 60

Avril 60

Juin 60

Octobre 60

Novembre 60 Janvier 61

Avril 61

Mai 61

Juin 61

Octobre 6I

Décembre 61

Mars 62

Avril 62

Septembre 62

Octobre 62
Les étudiants. . . . . . . . . . . . . . . 3,90 F

Le Canada français.. . . . . . . . . . . . . . . 5,50

La gauche américaine. . . . . . . . . . . . . . 3,90

Misère de la psychiatrie . . . . . . . . . . . . 5,50

Mensonges et vérités de nos anticipations. . . . 3,90

Pouvoir politique, pouvoir économique . . . . . 5,50

Situation de la peinture . . . . . . . . . . . . 3,90

Nos maisons et nos villes. . . . . . . . . . . . . . 5,50

Premières exigences d'une politique française. $\quad 3,90$

La réforme de l'enseignement . . . . . . . . . 5,50

Valeurs et limites de la Nation. . . . . . . . . 3,90

Le monde des prisons. . . . . . . . . . . . . . 5,50

L'Italie bouge. . . . . . . . . . . . . . . . . . . 5,50

Sciences de l'homme. . . . . . . . . . . . . . . 3,90

Les flammes de Budapest. . . . . . . . . . . . . . 3,90

Les médecins parlent de la médecine. . . . . . 5,50

Le temps de la réflexion. . . . . . . . . . . . . 3,90

Les maladies infantiles de l'indépendance. . . . 3,90

La France des Français . . . . . . . . . . . . 5,50

Politique et religion . . . . . . . . . . . . . 3,90

Jeunesse en Algérie. . . . . . . . . . . . . . . . 3,90

Le Nouveau Roman . . . . . . . . . . . . . . 5,50

Cinquième République?. . . . . . . . . . . . . 3,90

Amérique latine . . . . . . . . . . . . . . . . 5,50

Albert Béguin . . . . . . . . . . . 5,50

Les sciences sociales aux États-Unis . . . . . . 5,50

L'homme standard. . . . . . . . . . . . . . . 3,90

Pauvreté et monde moderne . . . . . . . . . . 3,90

La démocratie est une idée neuve. . . . . . . . . 3,90

Laïcité et paix scolaire . . . . . . . . . . . . . 399

Espagne vingt ans après. . . . . . . . . . . . . . . . . . . 3,90

Musique nouvelle. . . . . . . . . . . . . . . . 5,50

De la coexistence à la paix . . . . . . . . . . . 5,50

La lecture. . . . . . . . . . . . . . . . . . 3,90

Situation du cinéma français . . . . . . . . . . 5,50

L'obstacle de la propriété. . . . . . . . . . . . . . 3,90

La sexualité. . . . . . . . . . . . . . . . 7,00

Algérie, guerre et paix. . . . . . . . . . 4,50

Cuba, révolution menacée . . . . . . . . . . . 4,50

La femme au travail . . . . . . . . . . . . . . . . . . . . . . . 6,50

Marxisme et Christianisme. . . . . . . . . . . 3,90

De l'assistance à la solidarité. . . . . . . . . . 4,50

Vcux pour le Concile. . . . . . . . . . . . . . 6,50

Une nouvelle conscience politique . . . . . . . 4.40

Les Antilles, avant qu'il soit trop tard. . . . . . . 4,40

Les enseignants à la réforme. . . . . . . . . . 3,90

L'après-guerre d'Algérie . . . . . . . . . . . . . . . 5, 50

Vous pouvez commander ces numéros chez votre libraire ou à Esprit

19, rue Jacob, PARIS VI ${ }^{e}$ - C.C.P. Paris $1154-51$

POUR CONNAITRE NOS POSITIONS, LISEZ : ESPRIT « NOUVELLE SÉRIE 》

Novembre $1957: 3,90 \mathrm{~F}$ 


\section{études}

\section{RURALES}

REVUE TRIMESTRIELLE

D'HISTOIRE, GÉOGRAPHIE, SOCIOLOGIE

ET ÉCONOMIE DES CAMPAGNES

publiée par l'École pratique des Hautes Études

Sixième section - Sorbonne

Sommaire du No 8 (janvier-mars $\mathrm{I}_{96} 6_{3}$ )

P. Flatres. La deuxième « révolution agricole 》 en Finistère.

S. Mallet. A propos du syndicalisme agricole finistérien.

M. Gutrtelman. L'agriculture cubaine : la réforme agraire et les problèmes nouveaux.

Suivi de "Quelques commentaires» par R. Dumont.

J. Cujo. La consommation d'énergie dans le département de l'Hérault.

M. Carbonnell. Les formations végétales du Sud-Est de Madagascar.

Revue des revues

\section{Abonnement - 4 Numéros}

France et pays de la zone franc : $25 \mathrm{~F}$.

Autres pays : $28 \mathrm{~F}$. Le numéro : 7,50 $\mathrm{F}$.

Les abonnements peuvent être souscrits auprès de votre libraire habituel ou des Éditions MOUTON et Cie, s.a.r.l., 45 rue de Lille, Paris $7^{\mathrm{e}}$. C.C.P. Paris $50-6796$.

Rédaction : 20, rue de la Baume, Paris, 8 . 


\section{OFFENE}

Zeitschrift für Wirtschaft, Politik und Gesellschaft Herausgegeben vom Verein zur Förderung wirtschafts- und gesellschaftspolitischer Bildung in Zusammenarbeit mit der WirtschaftspoliWELT tischen Gesellschaft von 1947.

Schriftleitung : Dr. Dr. Gernot Gather, Frankfurt/Main.

Der Jahrgang umfaßt 4 Hefte im Umfang von je r 40 Seiten Format $14,5 \times 20,5 \mathrm{~cm}$. Der Einzelpreis des Heftes beträgt DM 3,50, der Abonnementspreis jährlich DM 12.

Jedes Heft der Offenen Welt ist einem besonderen wirtschafts-, sozial- oder kulturpolitischen Thema gewidmet, das unter den vielfältigen geistesgeschichtlichen, soziologischen, weltpolitischen und ökonomischen Aspekten dargestellt wird.

\section{Heft 74 (Dezember 1961) Weltbild in West und Ost}

Offene Gesellschaft, Die Revolution des Westens, Integrationsformen und Wirtschaftssysteme in West und Ost, Probleme des Fortschritts, Bildungskonzeptionen in West und Ost.

Heft 75 (März 1962) Unsere Kräfte in einer gefährdeten Welt Wortlaut der Referate von Petru Dumitriu, Waldemar Besson, Ralf Dahrendorf auf der zwölften Jahrestagung der Wipog am 19. Januar 1962 in Frankfurt am Main; Gerhard Schmidtchen : Die gesellschaftsbildende Kraft der Massenmedien.

Heft 76 (Juni 1962) Wachstumssorgen

Währungs- und konjunkturpolitische Probleme, Die Lohn- und Tarifpolitik in ihren volkswirtschaftlichen Zusammenhängen, Die Zukunft als Wille und Vorstellung, Strategie des Bildungswesens, Die Massenmedien umstritten und umworben.

\section{Heft 77 (September 1962) Das Weltagrarproblem}

Die Landwirtschaft in den Entwicklungsstufen der Gesellschaft, Gelten für die Landwirtschaft andere Produktionsgesetze? Problem und Verantwortung der europäischen Agrarpolitik, Agrarpolitische Leitbilder in West und Ost, Agrarprobleme in hochindustrialisierten und industriell schwachentwickelten Volkswirtschaften.

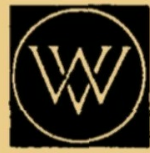

WESTDEUTSCHER VERLAG, KÖIN UND OPLADEN 


\section{Kölner Zeitschrift für Soziologie und Sozialpsychologie}

Begründet durch Leopold von Wiese, herausgegeben im Auftrage des Forschungsinstitutes für Sozial- und Verwaltungswissenschaften in Köln von Prof. Dr. René König, Köln.

Die unter Mitarbeit zahlreicher bekannter Soziologen von René König herausgegebene Viertelịahreszeitschrift gehört ohne Zweifel zu den besten soziologischen Publikationen Westdeutschlands, deren Lektüre auch für alle diejenigen lohnenswert ist, die nicht unmittelbar sich beruflich mit soziologischen Problemen zu befassen haben.

Besonders wird ein Psychologe wertvolle Anregungen bekommen, zumal seelenkundliches Bemühen von einer Erhellung der umgreifenden gesellschaftlichen Strukturen, der gesellschaftlichen Dynamik usw. untrennbar ist.

Forfa-Briefe

\section{BEZUGSBEDINGU NGEN:}

Jährlich erscheinen 4 Hefte (Format 16,5 $\times 24 \mathrm{~cm}$ ) mit zusammen 700 Seiten Umfang. Abonnementspreis jährlich DM 50,-, bei Vorauszahlung bis zum 1.2. des laufendèn Jahrgangs DM 45,- Einzelheft DM 14,50, zuzüglich Porto. Jährlich wird den Abonnenten ein Sonderheft zu einem Vorzugspreis geliefert. 


\section{KrKLOS}

Internationale Zeitschrift für Sozialwissenschaften

Revue internationale des sciences sociales

International Review for Social Sciences

\section{Editores : Lours Baudin, Paris}

Erwin von Beckerath, Bonn - Gottfried Bombach, Basel.

Howard S. Ellis, Berkeley - Alvin H. Hansen, Cambridge usa

Sir Roy F. Harroid, Oxford - J. R. Hicks, Oxford - W. A. Jöhr, St. Gallen

Frederic C. LANe, Baltimore - Wilhelm Röpke, Genève - Edgar Salin, Basel

Redactores : Jacques Stohler, Basel - Hans-Joerg ABt, Basel

$$
\text { Vol. XVI - } 1963-\text { Fasc. } 2
$$

EDGAR SALIN : Kartellverbot und Konzentration. . . . . .

J. H. Richter' : Toward an International Policy on Agricultural Trade.

F. G. Adams and L. E. Grayson : Economic Considerations of an Atlantic Energy Policy............

THEO SURANYI-UNGER : Strukturwandlungen der Investitionen in Südosteuropa .

MoRris Singer : Proposals Regarding Inflation without Full Employment in the US Economy .

Hernz KöHLer : East Germany's Terms of Trade. . . . . . A. GafNi, N. Halevi and G. Hanoch : Classification of Tariffs by Function . . . . . . . . . . .

\section{NOTES :}

Development, Mobility, and the Case for Tariffs - A Dissenting Note (P. B. Kenen) . . . . . . . . . . . . . The Threshold of 'Economic Growth (J. E. LA Touretre/R. BICANIC).

Besprechungen - Comptes rendus - Reviews . . . . . . . Bibliographie - Bibliography . . . . . . . . . . Autoren - Auteurs - Authors. 


\title{
L'HOMME \\ Revue francaise d'anthropologie
}

\author{
Comité de direction : \\ Émile Benveniste - Pierre Gourou \\ Claude Lévi-Strauss \\ Secrétaire général : J. Poulllon \\ Rédaction : Laboratoire d'Anthropologie Sociale, \\ 19, avenue d'Iéna, Paris xvie - Téléphone : KLE. 76-59.
}

Sommaire du $n^{0}$ I (mai-août 1963 ), tome III :

J.-L. Chambard

La Potbî du Jagâ ou le registre secret d'un généalogiste de village en Inde centrale.

Pertev N. Boratav

Un Motif de l'épopée oghouz.

Louis BERTHE

Morpho-syntaxe du Buna' (Timor central).

Notes et commentaires

Comptes rendus

Le numéro : fl. $6,75 / \$ 1,9 \% / t-13.6 / 9$ F. Abonnement pour trois numéros : fl. $18,75 / \$ 5,30 / \hbar$ 1.17.6/25 F. Les abonnements peuvent être souscrits auprès de l'administration, 20, rue de la Baume, Paris-VIII'. 


\title{
PREUVES revue mensuelle
}

\author{
En douze années \\ la revue \\ P R E U V E S \\ est devenue un véritable \\ Forum international \\ où se retrouvent chaque mois \\ des écrivains et des hommes politiques \\ s'adressant à des esprits libres \\ is \\ Par ses analyses et ses grands débats

$$
\text { PRE U VES }
$$ \\ prend une part capitale \\ à l'explication de notre temps
}

PREUVES a publié des essais de :

Raymond Aron - Roger Calliors - Albert Camus

T. S. Eliot - Pierre Emmanuel - Aldous Huxley

Karl JAspers - Arthur Kastrer - Herbert Luthy

S. de Madariaga - Vincent Monteil - Denis de Rougemont

Bertrand Russell - Ignazio Silone - Manes SpERber

Germaine Tillion - Georges VedeL, etc...

PREUVES, is avenue de l'Opéra - Paris (i er)

En vente en librairie

Spécimen gratuit sur demande 


\section{QUADERNI DI SOCIOLOGIA}

\section{Trimestrali}

\section{Comitato direttivo :}

Nicola Abbagnano, Franco Ferrarotti, Luciano Gallino, Angelo Pagani, Alessandro Pizzorno, Pietro Rossi, Tullio Tentori, Renato Treves.

Direttore responsabile : Franco Ferrarotti.

Redattore : Luciano Gallino.

\section{VOLUME XI - I962 - (NUOVA SERIE)}

\section{Fascicolo 3 (Luglio-Settembre)}

Talcotc Parsons Considerazioni teoriche intorno alla sociologia della medicina.

Renato Treves

La sociologia giuridica in Italia e i suoi possibili sviluppi.

Laura BALBO

Un aspetto dell'integrazione sociale degli immigrati in una grande città.

Pietro Rossi

La struttura della scienza e le scienze sociali.

Luciano SAFFirio

Appunti sull'attuale sociologia criminale.

Schede - Panorama riviste - Notiziario dell'A.I.S.S.

\section{Fascicolo 4 (Ottobre-Dicembre)}

Alberto M. Cirese Introduzione alla lettura di Sumner.

Luciano GALLINo L'organizzazione dell'azienda processiva.

Anne Parsons

Autorità patriarcale $\mathrm{e}$ autorità matriarcale nella famiglia napoletana.

Jean Meynaud A proposito della ricerca su sociologi e centri di potere in Italia.

Schede - Panorama riviste - Notiziario dell'A.I.S.S.

Abbonamento annu : per l'Italia L. 4000 , per l'estero L. 6000. Abbonamento sostenitore L. 20000.

Redazione e amministrazione : Casa editrice Taylor, via Valegigio 26, ToRINo. Telefono 584.15s - CC postale 2/34389. 


\section{REVUE FRANÇAISE $\mathrm{DE}$ \\ SOCIOLOGIE}

publiée par les soins du

CENTRE D'ÉTUDES SOCIOLOGIQUES

avec le concours $d u$

CENTRE NATIONAL DE LA RECHERCHE SCIENTIFIQUE

Directeur : J. StoetzeL

Vol. IV, $x$, janvier-mars ${ }_{96} 6_{3}$.

\section{ARTICLES}

Everett K. WiLson L'infiuence de Durkbeim aix Etats-Unis. Recherches empiriques sur le suicide.

Joffre Dumazedier Contenu culturel $d u$ loisir ouvrier dans six villes d'Europe.

Jacques MAITRE

David VICTOROFF

Représentation logaritbmique de phénomènes religieux.

Persuasion collective et image de soi.

CRITIQUE

Joffre DUMAZEDIER et Aline RIPERT

Où en est la sociologie du loisir et de la culture populaire?

ACTUALITÉ DE LA RECHERCHE

BIBLIOGRAPHIE

REVUE DES REVUES

Résumés des articles en anglais, allemand, espagnol, russe.

Direction, rédaction :

CENTRE D'ÉTUDES SOCIOLOGIQUES

82 , rue Cardinet,

PARIS (XVII).
Administration, abonnements :

JULLIARD

30-34, tue de l'Université,

PARIS (viro).

4 numéros de 128 pages, par an.

ABONNEMENT $\left\{\begin{array}{l}\text { France..... I } 8 \mathrm{~F} \text { (le numéro : } 5,10 \mathrm{~F}) \\ \text { ETranger... } 22 \mathrm{~F} \\ \text { U.S.A...... \$ } 4,50\end{array}\right.$ 


\section{PAST \&

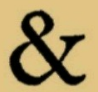

A journal of bistorical studies, founded 1952 Published April, July, November

Without any increase in price Past and Present changed from two to three issues a year in 1962 , and to meet still further demands on space the current issue (April 1963) has been increased to 104 pages, and includes :

KeItr Thomas : History and Antbropology.

W. T. Maccaffrey : Elizabethan Politics : The First Decade, I558-1568.

Philip Abrams : The Failure of Social Reform, 1918-1920.

Conference Report: Colonialism and Nationalism in Africa and Europe.

John F. H. New : Harrington, a Realist?

C. B. MACpherson : Harrington as a Realist : a Rejoinder.

Review articles, Communication, Reviews by Christopher Hill, R. H. Hilton, P. H. Sawyer, Lawrence Stone, S. S. Frere.

\section{Recent issues bave included:}

K. H. Connelu : The Potato in Ireland (Nov. 1962).

M. H. CuRTis : The Alienated Intellectuals of Early Stuart England (Nov. 1962).

Peter Marshall : Radicals, Conservatives and the American Revolution (Nov. 1962).

K. B. McFARLANe : England and the Hundred Years War (July 1962).

John Hale : War and Public Opinion in the Fifteenth and Sixteenth Centuries (July 1962).

M. I. FINLEY : Athenian Demagogues (April 1962).

Edouard Perroy : Social Mobility among the French Noblesse in the Later Middle Ages (April 1962).

John Elliotr : The Decline of Spain (Nov. 196r).

G. LEFF : Heresy and the Decline of the Medieval Cburch (Nov. 196r).

Full Table of Contents sent free on request.

All past issues which had been unobtainable for some time have now been reprinted and complete sets can once more be supplied.

Annual Subscription 15s (12 F). Current number gs (10 F)

Reduced Subscription for Students $\operatorname{ros}(7 \mathrm{~F})$

Past issues : $\operatorname{ros}(7 \mathrm{~F})$ per copy

Classified Table of Contents free on request

Write to : BUSINESS MANAGER, Past and Present,

Corpus Christi College, Oxford. England. 


\section{May 1963}

